\title{
Postoperative atrial fibrillation: Formulating the problem may be more challenging than the solution
}

\author{
Niv Ad, MD
}

\footnotetext{
From the Department of Cardiothoracic Surgery, West Virginia University School of Medicine, Morgantown, WVa.

Disclosures: Dr Ad is a consultant for Medtronic, a member of the speaker's bureau for AtriCure, proctor and member of the speaker's bureau for LivaNova, on the advisory board for Nido Surgical, and co-owner for Left Atrial Appendage Occlusion, LLC.

Received for publication Feb 17, 2017; accepted for publication Feb 28, 2017; available ahead of print March 29, 2017.

Address for reprints: Niv Ad, MD, Department of Cardiothoracic Surgery, West Virginia University School of Medicine, 1 Medical Center Drive, Morgantown, WV 26506 (E-mail: nivadmd14@gmail.com).

J Thorac Cardiovasc Surg 2017;153:1366-8

$0022-5223 / \$ 36.00$

Copyright (C) 2017 by The American Association for Thoracic Surgery

http://dx.doi.org/10.1016/j.jtcvs.2017.02.040
}

\begin{abstract}
The formulation of the problem is often more essential than its solution, which may be merely a matter of mathematical or experimental skill.
\end{abstract}

$$
\text { —Albert Einstein }
$$

Postoperative atrial fibrillation (POAF) is a common complication after cardiac surgery. The risk for POAF peaks at around 48 hours after surgery, but its incidence remains significant up to 7 days postsurgery. ${ }^{1}$ Historical rates suggest an incidence of POAF as high as 50\% in cases involving valve surgery but only up to $30 \%$ to $35 \%$ in more recent reports. ${ }^{2,3}$ The relatively lower incidence reported in recent studies and databases likely is related partly to broader use of perioperative beta blockers, prophylactic antiarrhythmic therapies, and better myocardial preservation in many centers. $^{4,5}$ The significance of POAF as reported by multiple studies is its association with perioperative complications, early and late mortality, and increased cost. $^{2}$ The ongoing challenge we have with the interpretation of these findings is related to the fact that many of the variables associated with inferior outcomes after cardiac surgery are very similar to those associated with POAF. Therefore, in many instances we should perhaps consider POAF as a marker for more complicated or high-risk patients and postoperative course rather than the sole cause for inferior outcomes.

In addition, more clarity is required in terms of the clinical significance of POAF. This should be based on the timing and the state of the patient at the time of the occurrence, true clinical impact in terms of hemodynamics and symptoms, duration, and the need for treatment, including anticoagulation. Regardless, societal efforts should be made to promote research to improve our understanding of the pathophysiology as well as gathering information on the most effective preventive and treatment protocols to reduce the incidence of POAF,

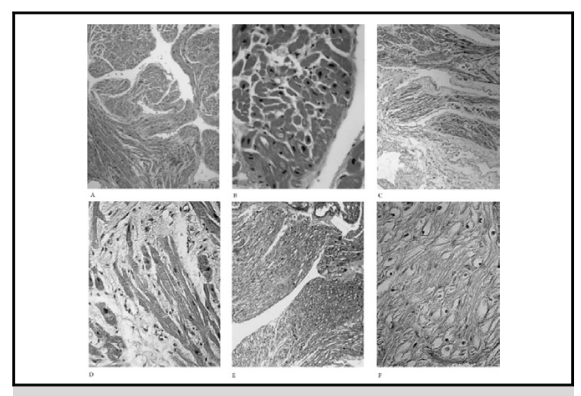

Ad N, Snir E, Vidne BA, Golomb E. Histologic atrial myolysis is associated with atrial fibrillation after cardiac operation. Ann Thorac Surg. 2001;72:688-93.

Central Message
Preexisting atrial substrate changes may
contribute to higher susceptibility to develop
postoperative atrial fibrillation following surgi-
cal manipulation and the associated inflamma-
tory response.

See Article page 1357.

even in the most complex patients. A better understanding of the real net impact of POAF on procedural cost is required, because current data available are not granular enough, and in most instances circumstantial evidence is being presented by the use of general billing tools that may include variables other than the cardiac surgery event only. ${ }^{2}$

When considering the potential etiologies associated with POAF, the multifactorial aspects of this complication should be recognized and understood. Irrespective of the etiology, POAF is an atrial arrhythmia and perhaps should be looked at as a final common pathway of multiple different pathologic events and clinical scenarios. The electrophysiologic aspects and basic mechanisms of POAF are less studied in humans but probably are a combination of single or multiple foci, single or multiple rotors, and reentry mechanisms that are directly related to operative stress, changes in myocardial properties, and surgical incisions. ${ }^{6}$

Clinical studies have defined a few common clinical variables associated with greater incidence of POAF, with the most consistent being age, chronic obstructive pulmonary disease, heart failure, obesity, renal failure, peripheral vascular disease, and possibly male sex. ${ }^{7,8}$ The role of perioperative inflammation was investigated in multiple studies, with conflicting evidence found regarding the efficacy of anti-inflammatory treatment 
and the impact on reduction in the incidence. ${ }^{9,10}$ The lack of consistency in the effect of anti-inflammatory treatment in general and more specifically steroids begs for a better understanding of the inflammatory process and its interaction with other clinical variables such as low cardiac output, poor oxygenation, use of inotropes, postoperative infection, and the specific patient's atrial substrate properties. ${ }^{11,12}$

Atrial substrate associated with greater susceptibility to develop POAF may have a more significant role than we previously thought. Research by others and us ${ }^{13-15}$ identified potential atrial myocardial properties that suggest greater susceptibility of developing POAF. Therefore, a better understanding of the role of preoperative atrial changes and potential markers for atrial substrate with greater vulnerability to develop POAF should be attempted. These studies recognized atrial myolysis, nuclear derangement, and atrial tissue aging as significant, and a better understanding of their role may improve efforts to control POAF and patient outcome. ${ }^{13}$ Mitochondrial dysfunction and poor response to simulated ischemic reperfusion injury also should be studied, because it may be possible to identify vulnerable atrial substrate very early after surgery so that better prophylactic strategies in reducing POAF could be applied. $^{1}$

In this issue of the Journal, Ishii and colleagues ${ }^{16}$ demonstrated the potential impact of a perioperative steroids regimen on attenuating the impact of the inflammatory response associated with right atrial atriotomy. A total of 18 mongrel dogs were divided to 3 groups of 6 dogs each. The first group was exposed to anesthesia only (control). The 2 other groups underwent right atriotomy, one without any treatment (atriotomy group) and the second with methylprednisolone treatment starting 1 week before the intervention and continued for 3 days after the intervention. A previous report by the authors also studied a fourth group that underwent pericariectomy only. ${ }^{17}$ The animal model is unique because it demonstrates the impact of surgery on the atrial myocardial properties and how such changes may be associated with acute response to surgery.

Interestingly, in their previous publications, ${ }^{17,18}$ the pericardiectomy-only group demonstrated significant changes in atrial tissue inhomogeneity index of atrial conduction, heart rate, and heart rate variability, changes that were found to be significant compared with the control and steroid-treated groups. This study also showed significantly greater inflammatory activity as measured by myeloperoxidase activity and neutrophil infiltration with a strong correlation between inhomogeneity in atrial conduction and the degree of inflammation. The degree of inhomogeneity of atrial conduction and inflammation correlated very well with the ability of inducing and maintaining atrial fibrillation (AF) longer than 2 minutes in the untreated atriotomy and the pericardiotomy group. Prophylactic treatment with steroids was found to be very effective in attenuating the inflammatory process and electrophysiologic changes. This impact was well correlated with lower rates of induction of AF by burst pacing and shorter duration of AF if induced for the atriotomy group treated with steroids and similar to the control group. ${ }^{16,17}$ In the current report, left atrial studies also were shared, which demonstrated very minimal impact on the left atrium.

This study is unique and important because it may indicate that inflammation has a primary role and impact on the measured changes and the electrophysiologic characteristics of the atrial tissue that may have led to greater tendency to develop and sustain AF after stimulation. The findings presented regarding the left atrium weaken to some extent the claim of inflammation as the only driver that is leading to these changes. Therefore, it also could suggest that the atriotomy and surgical manipulations of a specific atrial chamber are important and the inflammatory process seen is mainly the one that is associated with myocardial healing. It is well known that after myocardial injury there are 3 overlapping phases of healing: the inflammatory phase, in which inflammatory leucocytes are recruited and activated to clear the area of dead cells; the proliferative phase, in which suppression of proinflammatory signaling together with infiltration of cells secreting matrix proteins in the affected area; and the maturation phase. ${ }^{19}$ Therefore, one of the questions that should be asked is did the authors capture mainly an isolated inflammatory response or a mixture of the former and a response that is part of a physiological process that is associated with myocardial repair?

In their study in 2005, it clearly was demonstrated that inflammation is significant even in the pericadiectomyonly group with no atriotomy; however, it is important to remember that the right atrium was manipulated for mapping and that the pericardium adjacent to the right atrium was manipulated surgically. Both may result in increased inflammation. The studies clearly demonstrated that both the systemic and local inflammatory processes are important modifiers of atrial tissue properties and the ability to induce and sustain $\mathrm{AF}^{16,17}$ It would be imperative to better understand the potential implications of such findings with pathologic hearts, significant comorbid events, and the different atrial substrates.

There are a few important questions that can be raised and serve for future studies to further improve our understanding of POAF and help identify the best clinical approaches to reduce its incidence:

1. What is the impact of modification and attenuation of physiologic inflammatory response to myocardial injury on myocardial healing? The findings of the study clearly demonstrated that there is a significant inflammatory activity associated with a thoracotomy and the different 
atrial manipulation, such as atriotomy, perciariectomy, and intracardiac mapping. The clinical impact of high-dose periprocedural steroid treatment on myocardial healing and perioperative infections, however, is unknown and should be investigated for completeness.

2. In the clinical setting, it should be assumed that the physiologic inflammatory process exists in all patients and therefore an important research question should be why do only some patients develop POAF? I believe that this is a key question in which future studies could have the most impact. Are there patients with a stronger or a pathologic inflammatory process after an open heart surgery? Should we distinguish between tissue processes as part of the physiological response to injury and the systemic inflammatory response?

3. What are the preoperative clinical variables and atrial tissue substrate that are associated with greater susceptibility to develop POAF after tissue injury, ischemic events, and surgical stress? Research to address this question may help to improve patient screening and improve the efficacy of prophylactic treatment.

In summary, the findings of the study by the group from Washington University shed light on the association among atrial tissue properties, inflammatory response, surgical manipulation, and POAF. Future research should be focused on better understanding of the potential implications associated with differences in atrial substrate and the susceptibility of developing POAF.

\section{References}

1. Melby SJ, George JF, Picone DJ, Wallace JP, Davies JE, George DJ, et al. A timerelated parametric risk factor analysis for postoperative atrial fibrillation after heart surgery. J Thorac Cardiovasc Surg. 2015;149:886-92.

2. LaPar DJ, Speir AM, Crosby IK, Fonner E Jr, Brown M, Rich JB, et al. Postoperative atrial fibrillation significantly increases mortality, hospital readmission, and hospital costs. Ann Thorac Surg. 2014;98:527-33.

3. Society of Thoracic Surgeons. STS National Database. 2015. Available at: https://www.sts.org/national-database. Accessed 2015.
4. Davis EM, Packard KA, Hilleman DE. Pharmacologic prophylaxis of postoperative atrial fibrillation in patients undergoing cardiac surgery: beyond betablockers. Pharmacotherapy. 2010;30:749. 274e-318e.

5. Baker WL, White CM. Post-cardiothoracic surgery atrial fibrillation: a review of preventive strategies. Ann Pharmacother. 2007;41:587-98.

6. Nattel S. New ideas about atrial fibrillation 50 years on. Nature. 2002;415: 219-26.

7. Phan K, Ha HS, Phan S, Medi C, Thomas SP, Yan TD. New-onset atrial fibrillation following coronary bypass surgery predicts long-term mortality: a systematic review and meta-analysis. Eur J Cardiothorac Surg. 2015;48: 817-24.

8. Creswell LL, Schuessler RB, Rosenbloom M, Cox JL. Hazards of postoperative atrial arrhythmias. Ann Thorac Surg. 1993;56:539-49.

9. Zakkar M, Ascione R, James AF, Angelini GD, Suleiman MS. Inflammation, oxidative stress and postoperative atrial fibrillation in cardiac surgery. Pharmacol Ther. 2015;154:13-20.

10. Gaudino M, Andreotti F, Zamparelli R, Di Castelnuovo A, Nasso G, Burzotta F, et al. The $-174 \mathrm{G} / \mathrm{C}$ interleukin-6 polymorphism influences postoperative interleukin-6 levels and postoperative atrial fibrillation. Is atrial fibrillation an inflammatory complication? Circulation. 2003;108:II195-9.

11. Ho KM, Tan JA. Benefits and risks of corticosteroid prophylaxis in adult cardiac surgery: a dose-response meta-analysis. Circulation. 2009;119: 1853-66.

12. van Osch D, Dieleman JM, van Dijk D, Jacob KA, Kluin J, Doevendans PA, et al. DExamethasone for Cardiac Surgery (DECS) study group; DExamethasone for Cardiac Surgery DECS study group. Dexamethasone for the prevention of postoperative atrial fibrillation. Int J Cardiol. 2015;182:431-7.

13. Ad N, Snir E, Vidne BA, Golomb E. Histologic atrial myolysis is associated with atrial fibrillation after cardiac operation. Ann Thorac Surg. 2001;72:688-93.

14. Mariscalco G, Engström KG, Ferrarese S, Cozzi G, Bruno VD, Sessa F, et al. Relationship between atrial histopathology and atrial fibrillation after coronary bypass surgery. J Thorac Cardiovasc Surg. 2006;131:1364-72.

15. Ad N, Schneider A, Khaliulin I, Borman JB, Schwalb H. Impaired mitochondrial response to simulated ischemic injury as a predictor of the development of atrial fibrillation after cardiac surgery: in vitro study in human myocardium. $J$ Thorac Cardiovasc Surg. 2005; 129:41-5.

16. Ishii Y, Schuessler RB, Gaynor SL, Hames K, Damiano RJ Jr. Postoperative atrial fibrillation: the role of the inflammatory response. J Thorac Cardiovasc Surg. 2017:153:1357-65.

17. Ishii Y, Schuessler RB, Gaynor SL, Yamada K, Fu AS, Boineau JP, et al. Inflammation of atrium after cardiac surgery is associated with inhomogeneity of atrial conduction and atrial fibrillation. Circulation. 2005;111:2881-8.

18. Schuessler RB, Ishii Y, Khagi Y, Diabagate K, Boineau JP, Damiano RJ Jr. The effects of inflammation on heart rate and rhythm in a canine model of cardiac surgery. Heart Rhythm. 2012;9:432-9.

19. Bonaventura A, Montecucco F, Dallegri F. Cellular recruitment in myocardial ischaemia/reperfusion injury. Eur J Clin Invest. 2016;46:590-601. 